Supplemental Table 1a:

\begin{tabular}{|c|c|c|c|c|c|}
\hline \multicolumn{6}{|c|}{ PCR PRIMERS } \\
\hline Operon/ & Primer & & & & Prod. \\
\hline$\overline{\text { gene }}$ & $\overline{\text { Type }}$ & $\underline{\text { Strand }}$ & Primer Name & Sequence (5'-3') & $\overline{\text { Size }}$ \\
\hline sars & PCR & + & sarS-L & AAAGTGGGGCTTTGAATGTG & 1753 \\
\hline sarS & PCR & - & sarS-R & TGGGCGTACСTTATTCTTGC & \\
\hline rot & PCR & + & rot-L & CATGCTCCATTCATTTGTGC & 1501 \\
\hline rot & PCR & - & rot-R & АTTTAGCCTCATTCGGTTTG & \\
\hline rsb & PCR & + & rsb-L & TCGCGACATTTATGTGGATAC & 3673 \\
\hline rsb & PCR & - & rsb-R & GCTGAATGCAGTAGCTCACC & \\
\hline sarR & PCR & + & sarR-L & TTGCCTACACATGGTTTTGC & 1348 \\
\hline sarR & PCR & - & sarR-R & ATTTTGTGAGCAAGCCATCC & \\
\hline sarUT & PCR & + & sarUT-L & TGCTTCTGCCTCTTGTTGAG & 2424 \\
\hline sarUT & PCR & - & sarUT-R & AAGGTACGGCACCATCAAAC & \\
\hline agr & PCR & + & agr-L & AGATATCCTGCTCGCAGTGG & 4729 \\
\hline agr & PCR & - & agr-R & AAAATTGCGCCATAGGATTG & \\
\hline srr & PCR & + & srr-L & CGATGCTTCTTCGCAAATTC & 3476 \\
\hline srr & PCR & - & srr-R & АCTGTATGCGCTTTCCTGTG & \\
\hline sae & PCR & + & sae-L & TTCCGGGATATTTTTCAACG & 3974 \\
\hline sae & PCR & - & sae-R & TGCCCTCCTCTTATTTTGACTC & \\
\hline sarA & PCR & + & sarA -L & CGAACATTCAGGACATGCAC & 1375 \\
\hline sarA & PCR & - & sarA -R & AAACATTTAATTGCGCTAAATCG & \\
\hline spa (SA0095) & PCR & + & spaSA0095-L & TTGAGGCGTTTCAGAAGTTG & 2479 \\
\hline spa (SA0095) & PCR & - & spaSA0095-R & AAACCTCAGCACATTCAAAGC & \\
\hline $\mathrm{psm} \beta 1 \& 2$ & PCR & + & psm $\beta 1 \& 2-L$ & TCCAGCTGAGCTACCAGGAC & 1326 \\
\hline $\mathrm{psm} \beta 1 \& 2$ & PCR & - & $p s m \beta 1 \& 2-R$ & GTGAAACGCCCATTCTTCTG & \\
\hline psma1-4 & PCR & + & psma1-4 -L & ATTAGCAGAACGCCAAGACG & 2586 \\
\hline psma1-4 & PCR & - & psma1-4-R & TGTCATACCCCAGCAGAGTG & \\
\hline
\end{tabular}

Supplemental Table 1b:

\title{
SEQUENCING PRIMERS
}

\begin{tabular}{|c|c|c|c|c|}
\hline Operon & Primer Type & $\underline{\text { Strand }}$ & Primer Name & Sequence (5'-3') \\
\hline$\overline{\text { sarS }}$ & Sequencing & + & sarS-L1 & CGTTCTGCAATTTTCTCTCG \\
\hline sars & Sequencing & - & sarS-R1 & CAGATGATACCAAAAGATAG \\
\hline rot & Sequencing & + & rot-L1 & ATCCCAACAATCCCGAAAC \\
\hline rot & Sequencing & - & rot-R1 & GCGATTGCAAGTAGAGCAAC \\
\hline rsb & Sequencing & + & rsb-L1 & TCGAATCTATTTATGGCACC \\
\hline rsb & Sequencing & + & rsb-L2 & GCTTCACTAАCTGCAАTCTTGG \\
\hline rsb & Sequencing & + & rsb-L3 & CGAGTTCCATCTTGTCTCATAGG \\
\hline rsb & Sequencing & + & rsb-L4 & TCGTGTTTGTGAACTGATTCC \\
\hline rsb & Sequencing & + & rsb-L5 & CCACGATTAGTTGCCTCCTC \\
\hline rsb & Sequencing & - & rsb-R1 & GCGGCACAAAAAGTAAGTGG \\
\hline rsb & Sequencing & - & rsb-R2 & CATAAACATATGCACCCACAAG \\
\hline rsb & Sequencing & - & rsb-R3 & ACGGAGGTCGAATAACATGC \\
\hline rsb & Sequencing & - & rsb-R4 & GGCGAAAGAGTCGAAATCAG \\
\hline rsb & Sequencing & - & rsb-R5 & GCAATGGAAATGGGACAAAG \\
\hline rsb & Sequencing & - & rsb-R6 & CGAAGTTAAAGTCGGTGGAG \\
\hline sarR & Sequencing & + & sarR-L1 & ССТCTGATGCACATCTTGTTG \\
\hline sarR & Sequencing & - & sarR-R1 & GCTAAGTGCTCAGAGTTCAAACC \\
\hline sarUT & Sequencing & + & sarUT-L1 & TGСТСTTGCTTTTCAАATСАTTC \\
\hline
\end{tabular}




\begin{tabular}{|c|c|c|c|c|}
\hline sarUT & Sequencing & + & sarUT-L2 & TGAACGCCGTATTTTTGTTTC \\
\hline sarUT & Sequencing & + & sarUT-L3 & AGAACGATCCCTTGCAGATG \\
\hline sarUT & Sequencing & - & sarUT-R1 & AAAATACGGCGTTCATCTGC \\
\hline sarUT & Sequencing & - & sarUT-R2 & AAGGGATGAAGACTTGATGAATG \\
\hline sarUT & Sequencing & - & sarUT-R3 & TTCAATGCTATTGAGTTGATGG \\
\hline srr & Sequencing & + & srr-L1 & AACGCTGCAATAGGCTGAAC \\
\hline srr & Sequencing & + & srr-L2 & TCCATAАTATCATTCGCCATC \\
\hline srr & Sequencing & + & srr-L3 & TTCTGTGCTTTTGTTGGATAGC \\
\hline srr & Sequencing & + & srr-L4 & TATACCCAACGCCCCAGAC \\
\hline srr & Sequencing & - & srr-R1 & GAGCCGGCTAAATAGTGTCG \\
\hline srr & Sequencing & - & srr-R2 & CGTTTAAGAGACCAAGCTACACG \\
\hline srr & Sequencing & - & srr-R3 & CСАTTGTCCTTGATGAATCG \\
\hline sarA & Sequencing & + & sarA-L1 & САTCTTGCTCGATACATTTGC \\
\hline sarA & Sequencing & - & sarA-R1 & AGGAAGATAGCCGCATAACG \\
\hline spa & Sequencing & + & spa-L1 & GGCTTGTTATTGTCTTCCTC \\
\hline spa & Sequencing & + & spa-L2 & CATTTGCAGCAGGTGTTACG \\
\hline spa & Sequencing & + & spa-L3 & TTTTAGCTTCTGACAATAGG \\
\hline spa & Sequencing & - & spa-R1 & TGCTAACCTATTGTCAGAAG \\
\hline spa & Sequencing & - & spa-R2 & CAAACGGCACTACTGCTGAC \\
\hline $\mathrm{psm} \beta 1 \& 2$ & Sequencing & + & $\mathrm{psm} \beta-\mathrm{L} 1$ & CGCAAATACTGTGCAAGCTG \\
\hline $\mathrm{psm} \beta 1 \& 2$ & Sequencing & - & $\mathrm{psm} \beta-\mathrm{R} 1$ & TTTGTTTATCCGCACAACATC \\
\hline psma1-4 & Sequencing & + & $\mathrm{psm} \alpha-\mathrm{L} 1$ & САAAGCCACCАТСССТАТTG \\
\hline psma1-4 & Sequencing & + & psma-L2 & AATGGCCCССТTCAAATAAG \\
\hline psma1-4 & Sequencing & + & psma-L3 & AGGTCTCGTCTAGGCAAAGC \\
\hline psma1-4 & Sequencing & + & psm $\alpha-L 4$ & GGCTTTTTCGGTATTCATGG \\
\hline psma1-4 & Sequencing & - & psma-R1 & TGCTTTGCCTAGACGAGACC \\
\hline psma1-4 & Sequencing & - & psma-R2 & TTCACATGGGTATCATTGC \\
\hline psma1-4 & Sequencing & - & psma-R3 & TTGTGGCGTGTTTTATGTTTG \\
\hline psma1-4 & Sequencing & - & psma-R4 & TCAATTTCTTGCGCATTGAC \\
\hline sae & Sequencing & + & sae-L1 & CTGGGGGATATGTTTTACCTG \\
\hline sae & Sequencing & + & sae-L2 & САССАTTATCGGСTCСTTTC \\
\hline sae & Sequencing & + & sae-L3 & CGTAAAACGAGTTCCCTTGG \\
\hline sae & Sequencing & + & sae-L4 & АTTGCAATCTCTCCGAGTGG \\
\hline sae & Sequencing & + & sae-L5 & GGGGCTGTGAAATCATACG \\
\hline sae & Sequencing & + & sae-L6 & TСАTGСТААСТССТСАТTTСТTС \\
\hline sae & Sequencing & - & sae-R1 & СССАСТTACTGATCGTGGATG \\
\hline sae & Sequencing & - & sae-R2 & GATGCTAATACCGTGAATGTCC \\
\hline sae & Sequencing & - & sae-R3 & TTCTGAAGGACTACGTGATGG \\
\hline sae & Sequencing & - & sae-R4 & AGCTAATGAACTCGCGCAAC \\
\hline sae & Sequencing & - & sae-R5 & ССАTGAGCTCAААСАСТTCC \\
\hline agr & Sequencing & + & agr-L1 & CACAAATAAACTCGGATGAAGC \\
\hline agr & Sequencing & + & agr-L2 & САTTCGCGTTGCATTTATTG \\
\hline agr & Sequencing & + & agr-L4 & AAAAGAAGCCCATTCCTGTG \\
\hline agr & Sequencing & + & agr-L5 & CATTGGTAACATCGCAGCTTATAG \\
\hline agr & Sequencing & + & agr-L7 & TGTGCCATTGAAATCACTCC \\
\hline agr & Sequencing & + & agr-L8 & AGGGGCTCACGACCATAC \\
\hline agr & Sequencing & - & agr R1 & GAGGGCAATTTCCATAGGC \\
\hline agr & Sequencing & - & agr-R2 & TTTAGCTTGCTCAAGCACCTC \\
\hline agr & Sequencing & - & agr-R3 & GCTTCATCCGAGTTTATTTGTG \\
\hline agr & Sequencing & - & agr-R4 & TTGAATGAATTGGGCAAATG \\
\hline
\end{tabular}

